\title{
Surface viable counts with nichrome wire loops
}

\author{
OMAR KHAIRAT \\ From the Faculty of Medicine, Alexandria, Egypt
}

SYNOPSIS A surface viable counting method is described making use of a nichrome wire loop having an external diameter $5 \mathrm{~mm}$, which is streaked on an overdried plate. The loop holds $1 / 35 \mathrm{ml}(0.02857$ $\mathrm{ml}$ ) peptone water. When tried in parallel with pipettes that delivered $1 / 50 \mathrm{ml}(0.02 \mathrm{ml})$ drops, it gave reproducible results not at all inferior to those obtained with 50-dropping pipettes.

The introduction of Miles's surface counting method for viable bacteria with pipettes delivering $0.02 \mathrm{ml}$ drops (Miles and Misra, 1938) rendered the process of counting easier than by the pour-plate method. The purpose of the present paper is to describe a simple method of making viable counts, which is quicker because it demands no dropping pipettes and appears to be equally accurate. It consists in the use of calibrated nichrome wire loops to spread a known volume of diluted bacterial suspension on 'dried' agar or blood agar plates.

\section{Materials and Methods}

Nichrome wire $0.5 \mathrm{~mm}$ thick was used. The inoculating wire was made $13 \mathrm{~cm}$ or longer so that the holder did not touch the interior of the dilution tube. The widest possible loop that could hold a drop without letting it fall by gravity was selected. Loops having an external diameter of $6 \mathrm{~mm}$ were tried first but were found to be too wide. They were replaced by loops $5 \mathrm{~mm}$ wide. The diameter was checked with a measuring magnifier so as to make sure that it was $4 \mathrm{~mm}$ inside and $5 \mathrm{~mm}$ outside. The piston of a $1 \mathrm{ml}$ plastic disposable syringe (B.D. Plastipak) was found to be $4 \mathrm{~mm}$ in diameter. The small black piece at the nozzle end of the piston was removed and a pair of pliers helped to wind the nichrome wire around the piston. A nail or a metal rod $4 \mathrm{~mm}$ in diameter would also do. The particular magnifier used was the Bausch \& Lomb 'measuring magnifier' with a $20 \mathrm{~mm}$ scale in $1 \mathrm{~mm}$ divisions, but a microscope eyepiece with a micrometer disc having a scale $10 \mathrm{~mm}$ long dropped in could serve the purpose. In practice only three loops were

Received for publication 14 June 1974. required, since by the time the third had been used the first two had cooled.

In preparing dilutions, $4.5 \mathrm{mi}$ volumes of diluentusually peptone water- were put in test tubes, 150 $\times 15 \mathrm{~mm}$. Tenfold dilutions of an already diluted peptone water culture of $E$. coli were made. Volumes of $0.5 \mathrm{ml}$ were carried over from tube to tube by means of separate sterile straight-sided $1 \mathrm{ml}$ delivery pipettes graduated to the tip. Fluid was sucked up and down half the length of the pipette so that its inner surface became saturated with the bacteria that were going to cling to it and the bacterial aggregations were broken up. Excess culture fluid was removed from the outer surface of the tip of the pipette by touching the inside of the tube above the liquid before withdrawing (Report no. 71, 1956, 1969). When delivering the $0.5 \mathrm{ml}$ volume into a fresh tube care was taken never to dip the point of the pipette into the $4.5 \mathrm{ml}$ diluent, but to deliver the inoculum above it, then wait a few seconds for a little drop to collect near the tip, and blow out again (using the teat) before withdrawing. With a fresh sterile pipette the contents of the inoculated tube were forcibly mixed up and down 10 times before $0.5 \mathrm{ml}$ was carried to the next tube. A series of eight dilution tubes was sufficient. When the original culture was only faintly turbid, six tubes were enough. Before streaking the inocula the agar or blood agar plates were dried half opened, with the lids down, for one and half to two hours in a dry incubator at $37^{\circ} \mathrm{C}$.

To load the loop the culture tube was tilted to an angle of about 45 degrees, the loop introduced, dipped up and down the fluid 10 times to saturate the loop with the bacteria that would adhere to the wire, then suddenly jerked up. This loaded it with the maximum amount of fluid. Some experience was needed to judge that amount with the naked eye. The inoculating wire was then carefully withdrawn without touching the walls of the test tube and 
streaked along the overdried plate in the compartment reserved for it (fig). A plate with the diameter of the bottom dish measuring $9.4 \mathrm{~cm}$ accepted six streaks with comfort. When all the streaks were absorbed the plates were incubated, lids down, overnight. Six plates were a suitable number to average the counts, and a countable dilution, yielding some 15 to 60 colonies, was counted with ease in the $7 \times 1.2 \mathrm{~cm}$ rectangular space provided. Three dilutions could be streaked on half of each plate, making 12 counts on six plates.

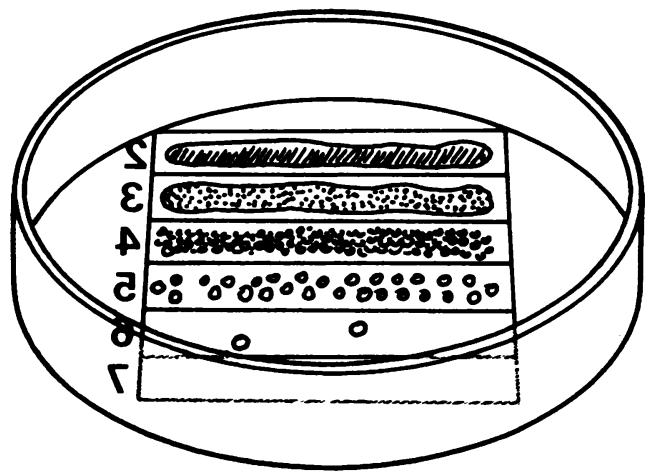

Fig. The culture dilutions six streaks on an agar plate. Numbers 2 to 7 denote $10^{-2}$ to $10^{-7}$.

$2=$ Confluent growth

$3 \& 4=$ discrete but uncountable colonies

The $10^{-5}$ dilution yielded countable colonies.

With the same dilutions, almost simultaneously, and with culture media poured from the same flask, counts were made by the Miles and Misra 50dropping pipettes so as to compare the results with the loop technique and determine the factor for the loop method. The two groups of plates were incubated for the same length of time before being counted.

\section{Results}

The table shows the results of the first two experiments done, one with the $6 \mathrm{~mm}$ loops and the other with $5 \mathrm{~mm}$ ones, on different days, both loops used on the same day of course. It will be noted that results with the $6 \mathrm{~mm}$ loop were as accurate as those with the drops, the $10^{-4}$ dilution yielding an average of 31 colonies whereas the $10^{-5}$ one grew an average of three. Experiments with the $5 \mathrm{~mm}$ loops were repeated a dozen times and gave similarly accurate counts every time.

The $5 \mathrm{~mm}$ loop was preferred to the $6 \mathrm{~mm}$ one because by the time the $6 \mathrm{~mm}$ loop came to the end of the track-a journey of $7 \mathrm{~cm}$-there was often a portion still undelivered and one had to streak backwards in the same compartment to release it. This did not happen with the $5 \mathrm{~mm}$ loop where the load was released except for a very thin membrane, so thin that it often ruptured by itself. In a loopful that would yield some 20 or more colonies the very thin film was unlikely to contain even one organism, and in any case the membrane was there every time.

To estimate the number of organisms per $\mathrm{ml}$ of the original culture, the average number of countable colonies growing from a $5 \mathrm{~mm}$ loopful are multiplied by the dilution used and by a factor 35 . If, for example, the countable dilution was $10^{-5}$ and the average colony count in six streaks 20 , then the number of viable organisms per $\mathrm{ml}$ of the original undiluted culture $=20 \times 10^{5} \times 35$. The factor 35 was arrived at by comparing the average number of colonies developing from $5 \mathrm{~mm}$ loopfuls of an appropriate culture dilution, to the average number of colonies growing from $0.02 \mathrm{ml}$ drops of the same culture dilution. For example in the table, at a dilution of $10^{-4}, 5 \mathrm{~mm}$ loopfuls yielded an average of 20 colonies compared with an average of 14 from $0.02 \mathrm{ml}$ drops, so the volume of a $5 \mathrm{~mm}$ loop was $1 / 35 \mathrm{ml}$.

Total Number of Colonies on Each Plate

\begin{tabular}{|c|c|c|c|c|c|c|c|c|c|c|c|c|c|c|c|}
\hline Experiment $^{1}$ & Inoculum from & Dilution Used & 1 & 2 & 3 & 4 & 5 & 6 & 7 & 8 & 9 & 10 & 11 & Total & Average \\
\hline \multirow{5}{*}{1} & $6 \mathrm{~mm}$ loop & $10^{-4}$ & 40 & 24 & 22 & 29 & 29 & 35 & 37 & 22 & 36 & 25 & 38 & 337 & 31 \\
\hline & $6 \mathrm{~mm}$ loop & $10^{-5}$ & 3 & 4 & 3 & 4 & 3 & 1 & 7 & 2 & 1 & 2 & 2 & 35 & 3 \\
\hline & $0.02 \mathrm{ml}$ & $10^{-4}$ & 7 & 10 & 11 & 13 & 11 & 10 & & & & & & 62 & 10 \\
\hline & $0.02 \mathrm{ml}$ & $10^{-5}$ & - & 1 & 2 & 2 & - & 1 & & & & & & 6 & 1 \\
\hline & $5 \mathrm{~mm}$ loop & $10^{-4}$ & 22 & 32 & 19 & 17 & 18 & 14 & 22 & 21 & 12 & 30 & 16 & 223 & 20 \\
\hline \multirow[t]{3}{*}{2} & $5 \mathrm{~mm}$ loop & $10^{-5}$ & 1 & 2 & 3 & 1 & 3 & 3 & 2 & 2 & 3 & - & 2 & 22 & 2 \\
\hline & $0.02 \mathrm{ml}$ & $10^{-4}$ & 17 & 13 & 12 & 16 & 19 & 8 & & & & & & 85 & 14 \\
\hline & $0.02 \mathrm{ml}$ & $10^{-5}$ & 1 & 4 & 1 & - & 2 & - & & & & & & 8 & 1 \\
\hline
\end{tabular}

Table The loop and drops methods performed in parallel

Experiments 1 and 2 were done on different days. 


\section{Discussion}

It can be said with confidence that the $5 \mathrm{~mm}$ loop does hold $1 / 35 \mathrm{ml}$ of peptone water. For other culture diluents such as broth or Ringer's solution these loops need not be recalibrated, but tested in parallel with $0.02 \mathrm{ml}$ volumes of culture dilutions from 50 dropping pipettes. In this case the factor $1 / 35$ may have to be altered.

In contrast to the dropping method where the size of the colony-bearing area was confined to a disc about $1.3 \mathrm{~cm}$ in diameter with the colonies crowded in such a small area, the inoculum with the loop method was spread along $7 \times 1.2 \mathrm{~cm}$, a rectangular area six times the area of the disc. Sometimes with the dropping method the falling drop jumped on the surface of the medium, split, and was deposited onto other sectors. This never occurred when the inoculum was spread with a loop touching the surface of the solid medium. Three loops take less than 10 minutes to make, whereas a battery of 50 or 60 pipettes takes two or three days. Moreover a headache-giving ticking metronome is not used. The tips of the dropping pipettes have to be checked with a micrometer screw gauge after the rough gauging with a perforated metal plate. The delivering stem of the dropper has to be some $9 \mathrm{~cm}$ long to render the sides of the nozzle as parallel as possible and not conical. After use the pipettes have to be disinfected in a suitable disinfectant solution or by moist heat. However carefully they are washed a proportion get their tips broken off. After washing, the pipettes have to be dried, plugged, and autoclaved before use. But the loops need only be flamed after use and are ready again for use in $\mathbf{2 0}$ seconds.

For a surface viable count with the $5 \mathrm{~mm}$ loops all that is needed is a series of tenfold dilutions of the culture, with the two or three dilutions expected to yield a countable number of colonies, eg, $10^{-4}, 10^{-5}$, and $10^{-6}$ streaked onto appropriate sectors of the overdried medium in six plates. With three dilutions streaked on each half plate and repeated on the other half, 12 counts could be made on the six plates, and even eight streaks could be made with care, thus economizing in agar and in plates. This reduces to a minimum any slight variation about the mean value.

The advantages of the loop technique certainly outweigh any slight variations about the mean value, so slight as to be scientifically irrelevant, as long as the higher dilution yields an average of exactly 10 times fewer colonies than the tenfold one above it.

\section{References}

Miles, A. A., and Misra, S. S. (1938). The estimation of the bactericidal power of the blood. J. Hyg. (Lond.), 38, 732-749.

Reports on Public Health and Medical Subjects, No. 71, London, 1956, 1969. 